PlastOx 2007 (2009) 147-159

(C) EDP Sciences, 2009

DOI: $10.1051 /$ ptox/2009011

\title{
Couplage hydrogène - plasticité dans les matériaux cubiques à faces centrées
}

\section{Delafosse}

\author{
École Nationale Supérieure des Mines de Saint-Étienne, PECM-UMR CNRS 5146, \\ 158 cours Fauriel, 42023 Saint-Étienne Cedex, France \\ e-mail : david.delafosse@emse.fr
}

\begin{abstract}
Résumé. Les interactions entre hydrogène en solution solide et plasticité sont étudiées et modélisées du point de vue de la métallurgie physique et de la théorie élastique des défauts discrets dans les cristaux cubiques à faces centrées. Des essais de vieillissement statique permettent d'évaluer quantitativement l'interaction élastique entre dislocations mobiles et atmosphères de solutés dans le nickel et dans un alliage binaire nickel-chrome. Les principes du couplage élastique entre solutés et dislocations sont rappelés et la notion d'indice d'écrantage des interactions de paires est introduite. Cette notion permet d'évaluer les effets de l'hydrogène sur l'énergie de ligne des dislocations, la tension de ligne, la contrainte critique d'activation de sources de Franck-Read, la stabilité des jonctions et la distance de séparation entre partielles qui gouverne le glissement dévié. Des essais de traction sur des monocristaux orientés pour le glissement simple permet d'évaluer les effets macroscopiques du couplage hydrogène - plasticité. L'observation par microscopie électronique à transmission des structures de déformation permet de mettre en évidence le glissement dévié comme étant le mécanisme élémentaire de plasticité le plus fortement affecté par la présence d'hydrogène en solution. Ces résultats sont discutés sous l'angle des mécanismes de rupture par effets de l'hydrogène.
\end{abstract}

\section{INTRODUCTION}

Les effets de l'hydrogène sur la déformation, l'endommagement et la rupture des métaux et alliages de structures sont multiples. Ils dépendent à la fois de la métallurgie (précipitation, recristallisation, état de surface), de l'activité de l'H et de son origine (H piégé lors de la solidification, $\mathrm{H}$ gazeux présent dans l'environnement et adsorbé, $\mathrm{H}$ issu de la réaction cathodique de réduction en corrosion aqueuse, ...) et du type de sollicitation mécanique (monotone/cyclique, plasticité localisée ou étendue ...). Les études expérimentales de ces phénomènes sont nombreuses. On pourra se reporter aux revues publiées sur ces phénoménologies [1-3]. Si la description des manifestations macroscopiques est bien établie, on ne dispose pourtant pas aujourd'hui d'un cadre général permettant de quantifier et prédire ces effets. Schématiquement, ils relèvent des catégories suivantes :

- Les décohésions inter-phases résultant de la recombinaison de l'hydrogène en solution en dihydrogène moléculaire et les effets de pression interne qui en résultent («blistering» des aciers par exemple)

- L'adsorption d'hydrogène monoatomique en fond de fissure de fatigue, qui affecte la réversibilité du glissement aux faibles amplitudes du facteur d'intensité des contraintes et le seuil de propagation en fatigue

- Les changement de phases induits par l'hydrogène et la déformation locale (précipitation dynamique d'hydrures fragiles en fond de fissure dans les alliages de zirconium, plus rarement de titane)

- Les ruptures fragiles inter- ou transgranulaires en Corrosion Sous Contrainte sous les effets combinés de la plasticité et de la ségrégation et du piégeage de l'hydrogène par les défauts de la cristallins ou microstructuraux.

En Corrosion Sous Contrainte, par exemple en milieu acide désaéré, l'activité de l'hydrogène associée à une surtension cathodique peut être très importante [4], ce qui conduit à des sursaturations importantes et très localisées de l'hydrogène en solution et à des interactions fortes avec les gradients locaux de 
contraintes et les défauts cristallins. Face à un problème d'endommagement assisté par l'H, la difficulté à identifier les mécanismes et quantifier les phénomènes mis en jeu a deux origines :

- Le caractère très localisé des phénomènes de couplage multi-physiques impliqués. L'échelle typique est celle du premier micromètre en fond de fissure. Cette zone peut s'étendre au premier grain ou joint de grains en pointe de fissure, rarement au delà. Analyser et modéliser ces effets implique donc de pouvoir déployer conjointement des moyens de modélisation micromécanique et des techniques de caractérisation microstructurale et d'analyse physico-chimique aux échelles les plus fines.

- En présence d'une telle activité de l'hydrogène, plusieurs des mécanismes précités peuvent opérer conjointement, en concurrence ou de manière synergique. C'est pourquoi les études de cas industriels de CSC assistée par l'hydrogène sont précédées par un important travail d'expertise visant à identifier et reproduire en laboratoire les mécanismes incriminés. A l'échelle d'une thèse de doctorat, ce travail indispensable peut s'avérer long et laisser une place insuffisante au développement et à la validation de modèles physiques d'endommagement.

Certains sous-problèmes ont cependant connu des avancées récentes significatives. Parmi ceux-ci, les mécanismes d'interactions entre l'hydrogène mobile en solution et la plasticité ont fait l'objet d'efforts particuliers, sous l'effet notamment de la popularité du modèle Hydrogen Enhanced Localised Plasticity (HELP) proposé il y a une vingtaine d'années par H. Birnbaum et l'équipe de l'université de l'Illinois [5]. Cet intérêt résulte de la conjonction entre un cadre théorique cohérent et des observation en microscopie à transmission in-situ sous pression partielle d'hydrogène dans un MET environnemental. La difficulté à tirer de ce modèle basé sur les interactions élastiques entre défauts cristallins des implications sur la réponse macroscopique - notamment celle des alliages c.f.c. - vient de la nature de la déformation plastique dans ces alliages, qui est intrinsèquement un mécanisme collectif ${ }^{1}$. Les interactions élastiques entre paires de dislocations ont une contribution marginale, ou du moins très indirecte, sur la réponse macroscopique. La tension de ligne, le franchissement de la forêt, ou les réactions mettant en jeu le glissement dévié jouent un rôle bien plus important dans les alliages austénitiques.

Nous illustrons ici ces points importants au travers d'une étude en trois parties sur les effets de l'hydrogène en solution solide sur la plasticité du nickel. Une étude expérimentale des phénomènes de vieillissement dynamique et statique couramment observés dans les solutions solides diluées et met en évidence de piégeage de l'hydrogène en solution interstitielle dans le champ élastique des dislocation et, indirectement, son transport par les dislocations mobiles. La seconde partie décrit la modélisation des interactions élastiques entre atmosphères d'hydrogène mobile et dislocations et ses conséquences sur les propriétés individuelles des dislocations, ainsi que certains mécanismes importants pour la plasticité. Nous présentons des résultats qui visent à introduire les effets élastiques de l'hydrogène mobile en solution dans un modèle simple de tension de ligne, à en évaluer les conséquences sur la multiplication des dislocations et la stabilité des jonctions. Ces résultats sont confrontés à une étude de la plasticité de monocristaux de Nickel pur et d'une solution solide désordonnée Ni-16Cr, basée sur des essais de déformation uniaxiale de monocristaux chargés en hydrogène et des observations par MET des microstructures de dislocations réalisées par X. Feaugas à l'Université de La Rochelle. Nous concluons sur les implications des phénomènes mis en évidence sur la déformation et la rupture des alliages c.f.c.

\section{2. ÉPINGLAGE DES DISLOCATIONS PAR L'HYDROGÈNE MOBILE : VIEILLISSEMENT STATIQUE}

Le premier niveau d'interaction entre hydrogène et plasticité est le piégeage et le transport de l'hydrogène mobile en solution dans le champ élastique des dislocations. Les expériences de désorption

\footnotetext{
1 Pour ajouter à la confusion, ce mécanisme est souvent improprement cité comme étant un mécanisme «d'adoucissement», alors qu'expérimentalement, la présence d'hydrogène en solution conduit presque systématiquement à une augmentation de la contrainte d'écoulement.
} 
du tritium en cours de déformation plastique et les observations par autoradiographie du tritium fournissent des preuves expérimentales directes de ces phénomènes [6]. Nous décrivons ici une approche expérimentale indirecte, mais plus simple de mise en œuvre. Elle est basée sur les phénomènes de vieillissement dynamique et statique qui apparaissent dans les solutions solides diluées lorsque les mobilités des dislocations et des atomes de soluté qui les épinglent sont du même ordre. Ce phénomène de vieillissement dynamique (Dynamic Strain Ageing : DSA) est bien documenté et bien modélisé. Sa manifestation la plus notable est l'apparition d'instabilités Portevin-Le Chatelier qui se traduisent par des chutes de force répétées sur les courbes de déformation et par des bandes de déformation périodiques sur les fûts d'éprouvettes lisses. Ce phénomène a été observé à des températures cryogéniques dans du nickel chargé en hydrogène par Wilcox et Smith [7]. Combette et Grilhé ont réalisé une étude complète des conditions d'apparition de ces instabilités, qui permet de les relier de manière claire au mécanisme de vieillissement dynamique [8]. Plus récemment, Girardin [9] a montré que le modèle à 2 densités de dislocations de Kubin et Estrin [10], dont les paramètres ont été identifiés par ses auteurs pour les alliages $\mathrm{Al}-\mathrm{Mg}$ autour de la température ambiante, pouvait être adapté au système nickel-hydrogène à basse température et utilisé pour interpréter quantitativement les résultats présentés ici.

Dans les alliages c.f.c., la friction de réseau est négligeable et on peut considérer que le mouvement des dislocations se fait par sauts instantanés entre rangées d'obstacles. Dans ces conditions, la loi d'Orowan peut s'écrire :

$$
\dot{\varepsilon}_{p}=\frac{b \rho_{m} \rho_{f}^{1 / 2}}{t_{a}}
$$

Avec $\rho_{m}$ et $\rho_{f}$ les densités de dislocation mobiles et de la forêt, $b$ la norme du vecteur de burgers des dislocations parfaites, $t_{a}$ le temps d'attente moyen entre deux sauts thermiquement activés ( $\rho_{f}^{1 / 2}$ représente le libre parcours moyen des mobiles entre deux rangées d'obsacles). Pendant leur temps d'attente entre deux sauts thermiquement activés, les dislocations immobilisées sont «vieillies » (épinglées) avec une cinétique exponentielle par les atomes de soluté mobiles :

$$
f\left(t_{v}\right)=f_{0}\left\{1-\exp \left[-\left(t_{v} / \tau\right)^{2 / 3}\right]\right\}
$$

$\tau$ est le temps caractéristique pour ce vieillissement et $f_{0}$ est la force d'épinglage à saturation $(\mathrm{t} \rightarrow \infty)$, lorsque la distribution d'atomes de soluté est à l'équilibre avec les gradients locaux de contrainte (la notion «d'équilibre avec les gradients de contrainte» est détaillée dans [11] et correspond à des temps de l'ordre de la milliseconde pour des alliages austénitiques à la température ambiante [12]). Le domaine de vieillissement dynamique et son éventuel sous-domaine d'existence des instabilités Portevin-Le Chatelier correspondent à un domaine dans lequel les temps caractéristiques d'attente $t_{a}$ et de vieillissement $t_{a}$ sont du même ordre. Dans ces conditions, toutes choses égales par ailleurs, une augmentation de la vitesse de déformation plastique se traduit, d'après Orowan eq. (1), par une diminution du temps d'attente. D'après (2), cette diminution entrâne une diminution de la force d'épinglage des dislocations par le nuage de solutés et donc de la contrainte d'écoulement. On est bien en présence d'une contribution négative de la sensibilité de la contrainte d'écoulement à la vitesse de déformation plastique, telle qu'elle est définie par la relation :

$$
S(\varepsilon)=\frac{\Delta \sigma}{\Delta \ln \left(\dot{\varepsilon}_{p}\right)}
$$

Pour une vitesse de déformation donnée, le minimum de $\mathrm{S}$ correspond donc au domaine de température dans lequel les temps d'attente et de vieillissement sont du même ordre. On peut, comme c'est souvent le cas, exprimer cette condition en affirmant que les «mobilités » des atomes de solutés et des dislocations sont semblables, à condition de garder à l'esprit le caractère discontinu du mouvement des dislocations et le fait que leur «mobilité » dans ce cas est une valeur apparente résultant du rapport entre le libre parcours moyen et le temps d'attente entre deux sauts instantanés. Dans le cas où la contribution de 


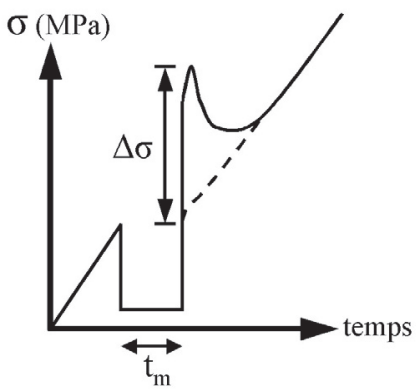

(a)

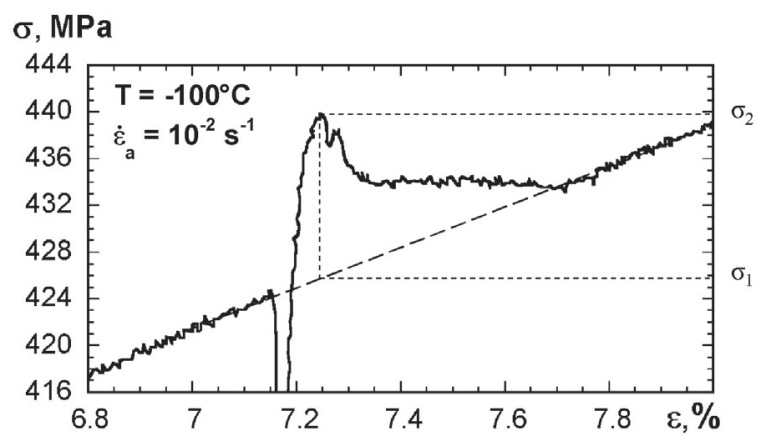

(b)

Figure 1. Essais de vieillissement statique. (a) Représentation schématique du transitoire à la recharge après un temps de maintien $\mathrm{t}_{m}$ à contrainte nulle. (b) mesure expérimentale de $\Delta \sigma=\sigma_{2}-\sigma_{2}$ dans $\mathrm{Ni}$ - $16 \mathrm{Cr}$ contenant $1600 \mathrm{ppm}$ d'hydrogène pour $\mathrm{t}_{m}=10 \mathrm{ha}-100^{\circ} \mathrm{C}$.

S à la contrainte d'écoulement est négative et suffisante en valeur absolue pour que la sensibilité totale soit négative, une augmentation locale de la vitesse de déformation abaisse la contrainte d'écoulement. Le système (échantillon + machine) est instable et on observe des systèmes plus ou moins organisés de bandes de déformation dont la dynamique dépend de la vitesse de déformation, de la température [13] et de la cinétique viscoplastique de relaxation des incompatibilités de déformation entre grains voisins [14]. En dehors du domaine de température / vitesse de déformation correspondant au creux de sensibilité de la contrainte d'écoulement à la vitesse de déformation (domaine du vieillissement dynamique), deux situations peuvent se présenter. Pour des températures supérieures et/ou des vitesses de déformation appliquées plus faibles, les atomes de soluté sont plus «mobiles » que les dislocations et celles-ci sont en permanence vieillies à saturation pendant leur temps d'attente : elles subissent une force d'épinglage $f_{0}$ eq. (2) par unité de longueur de ligne. A contrario, pour des températures plus basses et/ou des vitesses de déformation plus élevées, on peut considérer que le vieillissement est faible et que les dislocations se déplacent dans une solution solide interstitielle Nickel-Hydrogène pour le cas qui nous intéresse ici. C'est dans ce domaine de température que l'on peut évaluer la force d'épinglage. Si on stoppe brutalement la déformation et que l'on maintient l'échantillon sous une contrainte nulle ou faible pendant une durée $\mathrm{t}_{m}$, on impose $\mathrm{t}_{v} \approx \mathrm{t}_{a} \approx \mathrm{t}_{m}$ et on a ainsi un contrôle direct du temps de vieillissement. A la reprise de la déformation à la vitesse initiale, on observe un transitoire de la contrainte d'écoulement (figure 1) dont l'amplitude mesure une fraction de la force d'épinglage $f_{0}$. La fraction «manquante » correspond à une reprise de la multiplication des mobiles avant d'avoir atteint le pic de contrainte, la recharge n'étant pas instantanée.

Des polycristaux de nickel et d'alliage binaire $\mathrm{Ni}-16 \mathrm{Cr}$ ont été chargés en hydrogène par une exposition en autoclave de 48 heures entre 350 et $450^{\circ} \mathrm{C}$ sous une pression de 100 á 150 bars d'hydrogène gazeux. Le détail des conditions expérimentales est décrit dans [15]. Après refroidissement, on obtient une solution solide sursaturée contenant 1500 à $2000 \mathrm{ppm}$ atomique d'hydrogène et considérée comme homogène dans l'épaisseur des éprouvettes $(4 \mathrm{~mm})$. Dans le cas du Nickel, un essai de traction autour de $-70^{\circ} \mathrm{C}$ à une vitesse de déformation de $10^{-4} \mathrm{~s}^{-1}$ produit des instabilités Portevin-Le Chatelier. Suivant le raisonnement précédent, on réalise les essais de vieillissement statique à la même vitesse de déformation et à une température plus basse : $-140^{\circ} \mathrm{C}$ pour le Nickel. La même procédure dans l'alliage binaire $\mathrm{Ni}-16 \mathrm{Cr}$ permet de fixer la température d'essai à $-100^{\circ} \mathrm{C}$. Le déroulement des essais de vieillissement statique est le suivant :

- Après mise en précharge $\left(<50 \%\right.$ de $\left.\mathrm{Rp}_{0,2}\right)$ et stabilisation de la température, l'essai débute par une traction à vitesse de déformation imposée (10-4s-1), jusqu'à atteindre $7 \%$ de déformation plastique. Dans cette partie de l'essai, le temps d'attente des dislocations mobiles entre deux sauts 


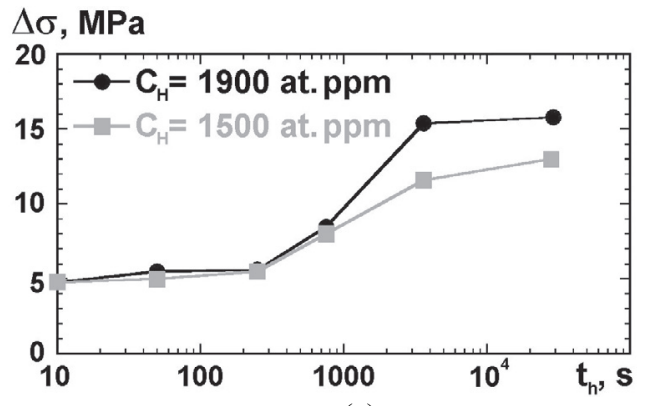

(a)

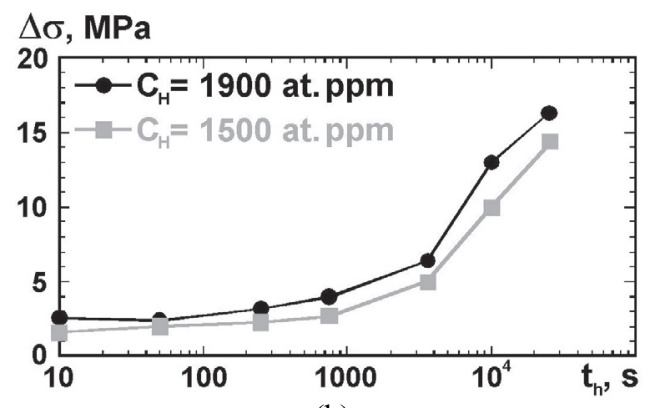

(b)

Figure 2. Pics de vieillisement statique $(\Delta \sigma)$ mesurés en fonction du temps de maintien $\mathrm{t}_{m}$. (a) à $-100^{\circ} \mathrm{C}$ dans $\mathrm{Ni}-16 \mathrm{Cr}$ et (b) à $-140^{\circ} \mathrm{C}$ dans $\mathrm{Ni}$, pour deux concentrations en hydrogène dissout.

thermiquement activés est fixé par la vitesse de déformation et par les densités de mobiles et de la forêt. Ce temps d'attente est insuffisant pour que les dislocations soient épinglées par l'hydrogène aux températures choisies $\left(-140^{\circ} \mathrm{C}\right.$ pour le nickel et $-100^{\circ} \mathrm{C}$ pour $\left.\mathrm{Ni}-16 \mathrm{Cr}\right)$

- A 7\% de déformation plastique, la machine passe en contrôle de charge. L'éprouvette est déchargée en dessous de $5 \mathrm{MPa}$ et maintenue à ce niveau pendant une durée qui varie entre 10 et $3 \cdot 10^{4} \mathrm{~s}$. Considérant qu'il n'ya pas de mouvement de dislocations significatif pendant ce maintien sous charge presque nulle, on assimile les temps de vieillissement $t_{v}$ et d'attente $t_{a}$ à ce temps de maintien $t_{h}$ imposé.

- L'essai reprend à la vitesse de déformation initiale. On enregistre alors un transitoire de la contrainte d'écoulement qui constitue la réponse du système au changement instantané de vitesse de déformation et de temps de vieillissement $\mathrm{t}_{\mathrm{v}}$. La mesure du pic de contrainte $\Delta \sigma$ associé est réalisée suivant la procédure préconisée par Combette et Grilhé [8] (figure 1a).

Les figure 2(a) et 2(b), donnent l'évolution du pic de contrainte $\Delta \sigma$ en fonction du temps de maintien pour les deux matériaux et les deux teneurs en hydrogène testées. Après un temps d'incubation le pic de contrainte associé au vieillissement augmente et sature avec le temps de maintien $\mathrm{t}_{\mathrm{m}}$. Ce comportement est qualitativement semblable à celui observé à plus haute température par De [16] dans un acier recuit à très bas carbone. La figure 2(b) correspond au vieillissement par l'hydrogène de l'alliage binaire $\mathrm{Ni} 16 \mathrm{Cr}$ à $-100^{\circ} \mathrm{C} . \Delta \sigma_{\text {sat }}$, la valeur saturée de $\Delta \sigma$, est atteinte pour des temps de vieillissement proches pour les deux concentrations en hydrogène, mais son amplitude est une fonction croissante de cette concentration. Pour $1900 \mathrm{ppm}$ atomiques, la saturation de $\Delta \sigma$ est atteinte après $\mathrm{t}_{\mathrm{m}}=10^{4}$ et vaut $16 \mathrm{MPa}$, contre $13 \mathrm{MPa}$ pour $1500 \mathrm{ppm}$ atomiques. Une adaptation du modèle de Kubin et Estrin à deux densités de dislocations pour simuler les transitoires de contrainte à la recharge permet d'estimer que $\Delta \sigma_{\text {sat }}$ mesure environ $80 \%$ de la force d'épinglage à saturation $f_{0}$ [9]. La fraction manquante correspond au désancrage précoce des dislocations pendant la recharge, avant d'avoir atteint le pic de contrainte $[10,15]$. La même évolution est observée dans le Nickel pur figure 2(a), mais décalée d'une décade vers des temps plus longs, du fait de la température d'essai plus basse. La saturation de $\Delta \sigma$ n'est pas atteinte après 8 heures de maintien (temps limité par la capacité du réservoir d'azote liquide dans notre installation), mais l'inflexion de $\Delta \sigma=f\left(t_{m}\right)$ est amorcée et on peut anticiper une valeur de $\Delta \sigma_{\text {sat }}$ de l'ordre de $20 \mathrm{MPa}$, plus élevée que dans l'alliage. De la même manière que dans le binaire, on constate qu'à tous les temps de maintien, $\Delta \sigma$ est une fonction croissante de la concentration en hydrogène entre 1500 et 1900 ppm. Aux températures auxquelles ces essais ont été réalisés, les pièges 'profonds' (cœurs des dislocations essentiellement) sont saturés pour des concentrations en hydrogène soluble bien inférieures à celles étudiées ici. On peut donc conclure que le supplément de contrainte d'épinglage observé entre 1500 et 1900 ppm de concentration moyenne correspond à un piégeage «faible » dans la composante hydrostatique du champ élastique des dislocations, tel qu'il est décrit dans la partie suivante. Autour de la température ambiante et pour des vitesses de déformation «ordinaires », les dislocations 
mobiles sont en permanence vieillies à saturation pendant leur temps d'attente sur les obstacles de la forêt. Ceci se traduit par une contribution positive constante à la contrainte d'écoulement de l'ordre de 10 à $20 \mathrm{MPa}$ suivant la concentration. Cette force «de traînage » des atmosphères de solutés a pu être mise en évidence lors d'essais de déformation sur des monocristaux chargés en hydrogène (cf. partie 4).

\section{MODÉLISATION DES INTERACTIONS ÉLASTIQUES HYDROGÈNE-PLASTICITÉ}

\subsection{Indice d'écrantage}

Les résultats précédents illustrent un premier niveau de couplage entre hydrogène et dislocations par piégeage des solutés dans le champ élastique des dislocations coin et nous avons mesuré la force d'épinglage associée. Un mécanisme de couplage en retour, prédit par l'élasticité linéaire, a été décrit de manière complète par Sofronis pour l'hydrogène en solution solide interstitielle [11], à partir du formalisme de Larché et Cahn [17]. Il résulte de la dilatation du réseau cristallin induite par la présence d'hydrogène en sursaturation dans le réseau et des profils de concentration en hydrogène dans le gradient de contrainte hydrostatique associé aux défauts cristallins. Le résultat net est un écrantage des interactions élastiques entre tous les défauts cristallins dont le champ élastique a une composante hydrostatique non-nulle. C'est le cas des interactions de paires entre dislocations dont le vecteur de Burgers a une composante coin non nulle, mais également, le cas échéant, des interactions dislocations / solutés non-mobiles ou dislocations / précipités. La théorie élasto-chimique de Larché et Cahn prédit également un couplage de second ordre qui conduit à une altération des constantes d'élasticité fonction de la concentration locale en soluté. Sofronis [18] propose une modélisation complète par éléments finis de ce phénomène pour l'hydrogène en solution solide autour de deux dislocations coin parallèles et montre que la prise en compte de l'effet sur les constantes d'élasticité affecte le champ élastique et les interactions de paires à courte portée (typiquement en dessous de 7 à 10 b, avec b le vecteur de Burgers). Considérant cet effet de second ordre comme négligeable en première approche, Chateau a proposé une modélisation alternative du couplage au premier ordre basée, sur une discrétisation du champ de concentration en solutés [19]. On montre ainsi que l'effet d'écrantage des interactions entre deux dislocations coins distantes de $\mathrm{d}$ a une dépendance en $1 / \mathrm{d}$ lorsque l'atmosphère de solutés est à l'équilibre avec les gradients locaux de contrainte. En conséquence, lorsqu'il est normalisé par les interactions de paires en l'absence d'hydrogène, l'effet d'un nuage d'atomes d'hydrogène en solution interstitielle est indépendant au premier ordre de la distance entre dislocations. On peut alors exploiter cette propriété pour définir un «indice d'écrantage » $\mathrm{S}$ qui quantifie l'effet relatif de l'atmosphère d'hydrogène sur les interactions de paires entre dislocations coin. En présence d'une concentration atomique moyenne $\mathrm{c}$ d'hydrogène mobile à l'équilibre avec les gradients locaux de contraintes, deux dislocations distantes de $\mathrm{d}$ et dont les composantes coin du vecteur de Burgers sont b1 et b2 respectivement exercent l'une sur l'autre une scission résolue qui vaut :

$$
\tau_{1 \leftrightarrow 2}=\frac{\mu b_{1} b_{2}(1-S(T, c))}{2 \pi(1-v) d}
$$

L'indice d'écrantage $\mathrm{S}$ dépend de la température absolue $T$ et de la concentration atomique $c$ :

$$
S(T, c)=\frac{S_{0}}{1+\beta \frac{T}{c}}
$$

$\beta$ étant fonction des constantes d'élasticité, du volume molaire de l'alliage et du volume molaire partiel de l'hydrogène en solution :

$$
\beta=\frac{9(1-v) R V_{M}}{2 E V^{* 2}}
$$

Avec $R$ la constante des gaz parfaits, $E$ le module d'Young, $v$ le coefficient de Poisson et $V_{M}$ le volume molaire de l'alliage, et $V^{*}$ le volume molaire partiel de l'hydrogène en solution solide interstitielle 
$\left(2 \mathrm{~cm}^{3} / \mathrm{mol}\right.$ dans les structures c.f.c.). Lorsque seuls les effets de dilatation isotrope du réseau sont pris en compte, on montre numériquement [19] que $S_{0}=0,75$, ce qui correspond à l'annulation complète du champ hydrostatique associé à une dislocation coin. Girardin a dérivé une solution analytique pour le champ de concentration à l'équilibre qui prend partiellement en compte la diminution du module de cisaillement en fonction de la concentration locale en soluté (couplage «au second ordre»). Dans ce cas, $S_{0}$ prend la forme [9] :

$$
S_{0}=\frac{1+v}{2}
$$

soit 0,67 pour le nickel. Cet indice d'écrantage, indépendant de la distance de séparation entre dislocations et le fait - démontré par les mesures de vieillissement statique - qu'à la température ambiante celles-ci sont en permanence vieillies à saturation entre deux sauts thermiquement activés peuvent être exploité pour exprimer une altération de la tension de ligne en présence de soluté, ainsi que les effets de cette concentration sur les propriétés des dislocations et mécanismes de plasticité faisant intervenir la tension de ligne. On trouvera le détail de ces calculs dans la thèse de Girardin [9]. Nous en donnons ici les principaux résultats.

\section{2 Énergie de ligne et tension de ligne}

Si on considère une dislocation initialement rectiligne et un cran infinitésimal le long de cette ligne, on peut évaluer l'incrément d'énergie de ligne associé à cet incrément de longueur. Considérant le modèle de De Wit et Koehler [20], l'énergie de ligne par unité de longueur dépend du caractère $\theta$ de la dislocation, mesuré comme l'angle entre le vecteur de Burgers et la ligne de dislocation (écart angulaire au caractère vis). En intégrant les interactions élastiques entre les segments élémentaires constituant la ligne, on obtient l'énergie de ligne par unité de longueur :

$$
E(\theta)=\frac{\mu b^{2}}{4 \pi} K(\theta) \ln \left(\frac{R}{r_{0}}\right) \quad \text { avec } \quad K(\theta)=\frac{1-v \cos ^{2} \theta}{1-v}
$$

où $b$ est la norme du vecteur de Burgers, $\mu$ le module de cisaillement, $v$ le coefficient de Poisson, $R$ et $r_{0}$ les rayons de coupure respectivement extérieur et intérieur (bornes d'intégration pour le calcul de l'énergie élastique autour de la dislocation). En présence d'hydrogène à l'équilibre avec les gradients locaux de contrainte, on introduit l'indice d'écrantage $S(T, c)$ défini par la relation (5) et $\mathrm{K}(\theta)$ devient :

$$
K^{H}(\theta)=\frac{1-S(T, c)-(v-S(T, c)) \cos ^{2} \theta}{1-v}
$$

La variation de $K$ en fonction de $\theta$ est tracée figure 3(a) pour trois valeurs de l'indice $\mathrm{S}$ (11, 22 et $33 \%$ ) qui correspondent à des concentrations atomiques respectives de $1,2 \cdot 10^{-2}, 2,9 \cdot 10^{-2}$ et $5,5 \cdot 10^{-2}$ dans le nickel à la température ambiante. En relaxant la composante hydrostatique du tenseur des contraintes, l'hydrogène en solution abaisse l'énergie de ligne des segments coin $\left(\theta=90^{\circ}\right)$ et n'affecte pas les parties vis $\left(\theta=0^{\circ}\right)$ au premier ordre.

Dans le modèle de De Wit et Koehler, la tension de ligne est définie comme la dérivée de l'énergie de ligne par rapport à sa longueur lors d'une déformation incrémentale. On obtient l'expression suivante pour la tension de ligne en présence d'hydrogène :

$$
\zeta^{H}(\theta)=\frac{\mu b^{2}}{4 \pi(1-v)}\left[1-2 v+S(T, c)+3 \cos ^{2} \theta(v-S(T, c))\right] \ln \left(\frac{R}{r_{0}}\right)
$$

La variation en fonction de $\theta$ est tracée figure 4(b) pour trois valeurs de $S(T, c)$. La tension de ligne des segments vis est abaissée alors que les parties coin sont rendues plus rigides. 


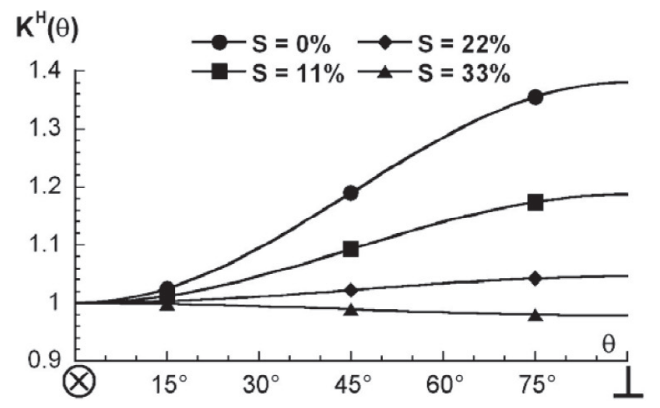

(a)

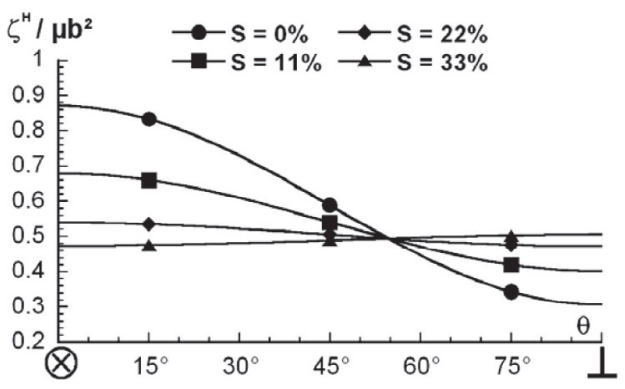

(b)

Figure 3. (a) Évolution de $K^{H}(\theta)$ et (b) de la tension de ligne $\zeta^{H}(\theta)$ en fonction du caractère de la dislocation pour différentes valeurs du taux d'écrantage dans le cas du nickel $\left(R / r_{0}=500\right)$.

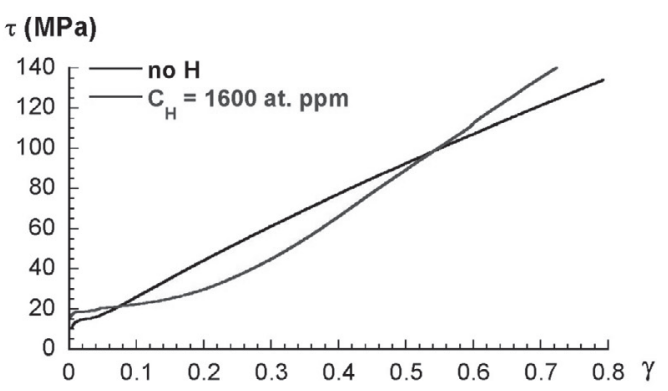

(a)

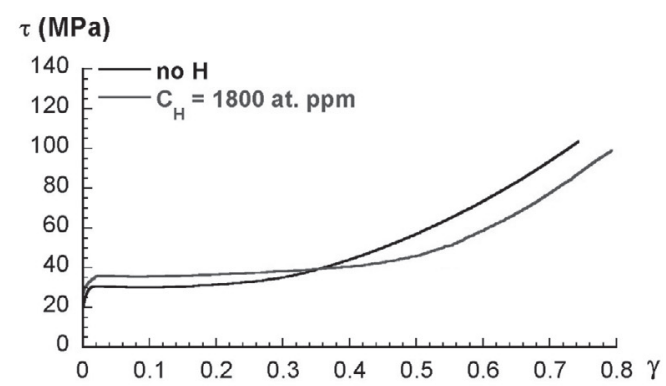

(b)

Figure 4. Courbes contrainte - deformation des monocristaux de nickel (a) et $\mathrm{Ni}-16 \mathrm{Cr}$ (b) chargés ou non en hydrogène.

\subsection{Expansion isotrope d'une boucle (mécanisme de Frank-Read)}

A partir des résultats précédents, on peut évaluer l'effet de l'hydrogène en solution sur la contrainte critique pour l'expansion d'une boucle isotrope. Reprenant l'approximation de Hirth et Lothe d'une boucle circulaire par un hexagone de coté $L$, nous exprimons l'énergie totale de la boucle hexagonale par la relation:

$$
W^{H}(L)=\frac{3 \mu b^{2} L}{4 \pi(1-v)}\left[0,16(2-v-S(T, c))+\left(2-v-S(T, c) \ln \left(\frac{R}{r_{0}}\right)\right]\right.
$$

La force critique pour l'expansion isotrope d'une telle boucle correspond à la dérivée première de cette énergie par rapport au «rayon» $L$. Comme pour les interactions de paires, on peut normaliser cette force en présence d'hydrogène $F^{H}$ par sa valeur en l'absence de solutés $F$, ce qui donne la valeur sans dimension :

$$
\frac{F^{H}}{F} \approx 1-0,58 \cdot S(T, c)
$$

En présence d'hydrogène en solution solide, la multiplication à partir de sources existantes est facilitée par l'abaissement de l'énergie de ligne des boucles de dislocation et, du fait de l'écrantage des interactions de paires, l'exhaustion de ces sources par effet de «back stress » est retardée. 


\subsection{Glissement dévié}

Dans les cristaux c.f.c., une dislocation parfaite de vecteur de Burgers $b=1 / 2\langle 110\rangle$ et de type $\theta$ se dissocie en deux partielles de Shockley de vecteur de Burgers $b_{p}=1 / 6\langle 112\rangle$ et de type $\theta \pm \pi / 6$ séparées par un ruban de faute d'empilement dont l'énergie par unité de surface vaut $\Gamma$. En l'absence de force extérieure, les partielles sont séparées l'une de l'autre d'une distance $d_{0}$, distance à laquelle deux forces s'équilibrent : la répulsion due à l'autre partielle $\left(\alpha 1 / d_{0}\right)$ et une force attractive constante qui dérive de l'énergie de défaut d'empilement $\Gamma$, propriété de l'alliage considéré. En présence d'hydrogène, la distance de séparation $d_{0}$ devient $d^{H}$ et s'écrit :

$$
d^{H}=\frac{\mu b_{p}^{2}}{8 \pi \Gamma} \frac{2(1-S(T, c))-(v-S(T, c))(1+2 \cos 2 \theta)}{1-v}
$$

Considérant une dislocation vis $\left(\theta=0^{\circ}\right)$, la distance de dissociation sera accrue en présence d'hydrogène $(1>\mathrm{S}>0)$, ainsi que le travail nécessaire pour la constriction et la recombinaison des partielles en dislocation parfaite, processus thermiquement activé nécessaire pour le glissement dévié. Ceci vient s'ajouter à un effet de l'hydrogène sur l'abaissement de $\Gamma$, ainsi qu'à un abaissement probable de la position de col (énergie d'activation) pour la déviation des segments vis [21]. Au final, la présence d'hydrogène en solution réduit significativement la probabilité de glissement dévié, par une combinaison d'effets de cœur et d'effets élastiques.

\section{DÉFORMATION UNIAXIALE DE MONOCRISTAUX}

Nous illustrons les résultats précédents en comparant les courbes de déformation de monocristaux de nickel pur et d'alliage binaire $\mathrm{Ni}-16 \mathrm{Cr}$ orientés pour glissement simple, contenant ou non de l'hydrogène en solution solide. Des éprouvettes prismatiques de section carrée $(4 \mathrm{~mm}$ de coté, $10 \mathrm{~mm}$ de longueur utile) sont préparés et chargés en hydrogène suivant la même procédure que dans l'étude du vieillissement statique. La concentration en hydrogène des monocristaux de nickel après chargement est de $1600 \mathrm{ppm}$ atomiques. La figure 4a) donne les courbes de traction du nickel chargé ou non, pour une vitesse de déformation de $10^{-5} \mathrm{~s}^{-1}$. On remarque d'emblée que vouloir rationnaliser les effets de l'hydrogène en termes «d'adoucissement» ou de «durcissement» La comparaison des deux courbes donne les informations suivantes :

- L'hydrogène entraine une augmentation faible mais distincte de la contrainte critique d'écoulement $\tau_{c}\left(\sim 5 \cdot 10^{-5} \mu\right.$, soit $10 \mathrm{MPa}$ en traction). Ceci correspond à la contrainte de traînage des atmosphères de solutés : nous avons vu qu'à la température ambiante et pour des vitesses de déformation «usuelles », les dislocations mobiles étaient vieillies à saturation par l'hydrogène mobile et que les valeurs d'épinglage dans le nickel polycristallin étaient de l'ordre de $15 \mathrm{MPa}$ pour $1900 \mathrm{ppm}$ atomiques d'hydrogène en solution.

- L'hydrogène retarde la transition stade I/stade II. La contrainte d'écoulement dans le stade I («glissement facile») est celle nécessaire pour entretenir la multiplication à partir des sources du système primaire. L'auto-écrouissage de ce système résulte d'interactions élastiques entre dipôles et de la formation et du stockage de jonctions colinéaires. La stabilité des jonctions entre dislocations peut être évaluée à partir d'un calcul d'équilibre des tensions de ligne entre les segments qui forment les point triples délimitant les jonctions. Un calcul de stabilité de ces jonctions en présence d'hydrogène prenant en compte les effets sur la tension de ligne (eq. (10)) montre que les jonctions de type Hirth et colinéaire sont celles dont le domaine d'existence et la stabilité sont les plus affectées par l'hydrogène en solution [9]. Ceci a pour effet de limiter l'auto-écrouissage du système primaire et donc de retarder indirectement l'activation des sources des systèmes secondaires, moins favorablement orientées, donc le début du stade II.

- Dans le stade II, la présence d'hydrogène en solution dans le nickel entrâne une augmentation significative du taux d'écrouissage. Ce stade d'écrouissage linéaire rapide est caractérisé par 


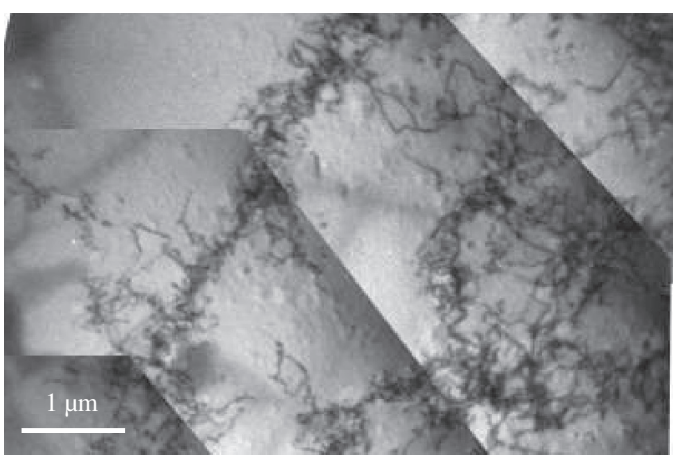

(a)

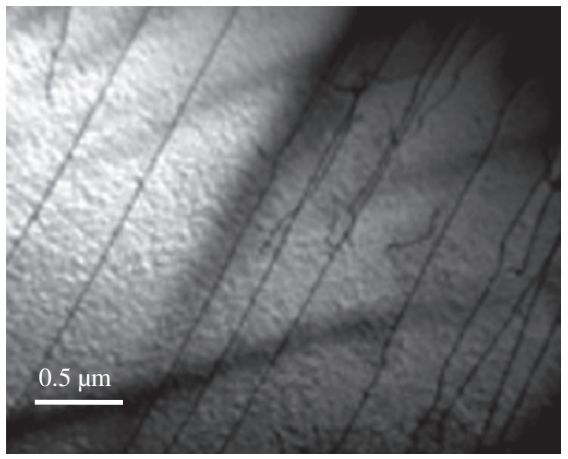

(b)

Figure 5. Observation en MET des structures de dislocation dans le plan [153] de monocristaux (a) de nickel et (b) de nickel chargé en hydrogène ( « Ni-H») après une déformation en cisaillement de 0,2 . Le monocristal de nickel est en stade II et on observe les précurseurs de la formation de cellules, alors que dans Ni-H, une faible densité de lignes vis parallèles, typique du stade I, est observée.

l'activation des systèmes secondaires et le taux de durcissement dépend de l'évolution du libre parcours moyen des dislocations mobiles. Ce paramètre est étudié dans la suite par microcopie électronique à transmission.

${ }^{2}$ Compte tenu des conditions de préparation des échantillons et de chargement en hydrogène, ces résultats fournissent des preuves expérimentales directes des effets de l'hydrogène sur les mécanismes de déformation des cristaux c.f.c. Des observations comparables sont obtenues dans des monocristaux d'alliage binaire $\mathrm{Ni}-16 \mathrm{Cr}$ figure (4b) et, qualitativement, dans des monocristaux d'acier inoxydable austénitique $\mathrm{Fe}-18 \mathrm{Cr}-16 \mathrm{Ni}-10 \mathrm{Mn}$ chargés électrolytiquement par Yagodzinskyy et al. jusqu'à des concentrations environ 7 fois plus élévées [22]. On note dans l'alliage binaire Ni-16Cr l'apparition d'un phénomène de «pseudo-Lüders» en début de stade I (durcissement d'écrouissage nul, mais ne donnant pas naissance à des bandes de déformation macroscopiques), alors que des bandes de Lüders très marquées ont été observées dans les monocristaux Fe-18Cr-16Ni-10Mn de Yagodzinskyy et collaborateurs contenant 7000 ppm. atomiques d'hydrogène.

La comparaison par Microscopie Electronique à Transmission (MET) des microstructures de déformation obtenues dans $\mathrm{Ni}$ et $\mathrm{Ni}-\mathrm{H}$ a été effectuée par X. Feaugas au LEMMA et fournit des indications précieuses sur les mécanismes affectés par l'hydrogène. Des lames minces ont été prélevées dans des échantillons de $\mathrm{Ni}$ et $\mathrm{Ni}-\mathrm{H}$ après des cisaillements plastiques de 0,2 et 0,7 , soit après que les courbes contrainte-déformation de sont croisées figure 4(a). On compare donc entre elles des microstructures qui présentent la même contrainte d'écoulement, mais des taux de durcissement différents. La figure 5 montre les microstructures à $\gamma=0,2$. L'échantillon hydrogéné est encore nettement en stade I. La microstructure est essentiellement composée de longs segments vis parallèles entre eux régulièrement décorés de (doubles) décrochements résultant de glissements déviés figure 5(b). La densité moyenne de dislocations est de $\rho=5 \cdot 10^{13} \mathrm{~m}^{-2}$. Le caractère majoritairement vis des lignes semble contredire ce qui pourrait être prédit à partir de l'équation (10). Il faut garder à l'esprit que sur la figure 3, seules les concentrations les plus élevées en hydrogène permettent à l'énergie de ligne des segments coin de passer en dessous de celle des vis. Pour le même niveau de déformation, le nickel pur est en début de stade II et on observe des cellules en cours de formation figure 5(a). La densité moyenne reste cependant légèrement inférieure à celle du nickel hydrogéné à cette déformation $\left(\rho=3 \cdot 10^{13} \mathrm{~m}^{-2}\right)$. A $\gamma=0,7$ la microstructure de cellules équiaxes est bien formée dans le nickel pur, ce qui témoigne d'une entrée dans le stade III et d'une forte activité du glissement dévié figure 6(a). Les murs de type GND (dislocations géométriquement nécessaires) sont espacés de 3 à $4 \mu \mathrm{m}$. L'échantillon hydrogéné 


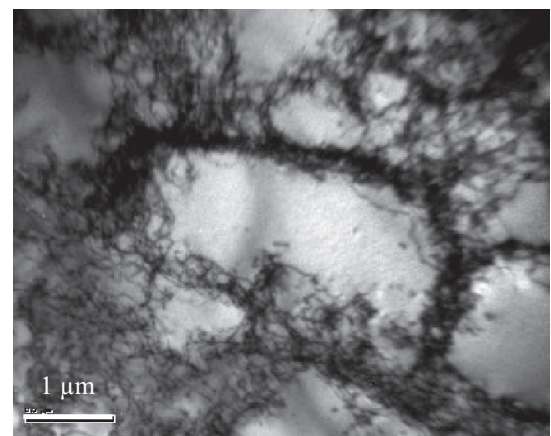

(a)

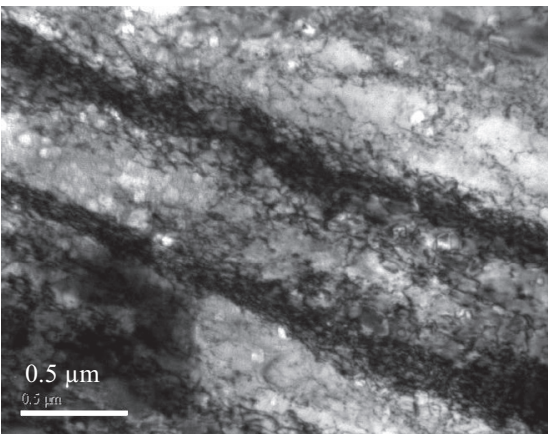

(b)

Figure 6. Observation en MET des structures de dislocation dans le plan [153] de monocristaux (a) de nickel et (b) de nickel chargé en hydrogène $(« \mathrm{Ni}-\mathrm{H} »)$ après une déformation en cisaillement de 0,7 . Le monocristal de nickel est nettement en stade III avec des cellules équiaxes bien établies, alors que dans $\mathrm{Ni}-\mathrm{H}$, une forte densité de murs parallèles, typique du stade II, est observée.

présente quant-à-lui une structure de murs parallèles à un plan $\{111\}$ typique du stade II et d'alliages à faible Energie de Défaut d'Empilement (EDE). L'espacement entre murs de GND est de l'ordre de 0,5 $\mu \mathrm{m}$. Ce libre parcours moyen fortement abaissé est à l'origine du durcissement plus important dans cette structure figure 6(b). On constate donc que parmi les mécanismes élémentaires de plasticité affectés par l'hydrogène mobile en solution interstitielle, le glissement dévié est celui qui est le plus affecté et celui via lequel les conséquences sur la contrainte d'écoulement sont, bien qu'indirectes, les plus importantes.

\section{CONCLUSIONS}

Cet ensemble de travaux expérimentaux et de modélisation montrent que les interactions entre hydrogène mobile en solution et déformation plastique peuvent être efficacement étudiés à l'aide de la «boîte à outils» traditionnelle de la métallurgie physique. Les essais de vieillissement statique constituent une approche macroscopique remarquablement efficace et relativement simple de mise en œuvre pour évaluer quantitativement l'interaction dynamique entre hydrogène et dislocations mobiles. Le formalisme «hydrogen elasticity» de Sofronis peut être introduit dans les expression classiques des propriétés des dislocations, sous réserve que celles-ci soient correctement décrites par la théorie élastique des défauts discrets. Les propriétés qui dérivent de la structure de cœur des dislocations, telles que par exemple la position de col donnant l'énergie d'activation du glissement dévié, doivent être approchées par des méthodes atomistiques en vue de dériver des modèles simplifiés utilisables à l'échelle des dislocations discrètes. La théorie élastique des dislocations permet d'évaluer quantitativement les effets de l'hydrogène sur les mécanismes de plasticité qui dépendent principalement de l'énergie élastique, de la tension de ligne et des interactions de paires. La multiplication ainsi que la formation et la destruction de jonctions sont aux nombre de ceux-ci. Malheureusement, l'étude expérimentale de la déformation des monocristaux montre que parmi les mécanismes élémentaires de la plasticité des cristaux c.f.c. affectés par l'hydrogène en solution solide, c'est le glissement dévié qui a les conséquences pratiques les plus importantes sur la réponse macroscopique. Ce mécanisme est un «mélange » d'effets élastiques et d'effets de cœur. Une modélisation prédictive sur des bases physiques des effets de l'hydrogène sur le glissement dévié constitue donc un problème multi-échelles qui reste à traiter. Il est par ailleurs notable que, même dans des alliages à faible énergie de faute (aciers inoxydables austénitiques par exemple), la présence d'hydrogène en solution de traduit par une localisation accrue du glissement. De nombreuses observations qualitatives de ce phénomène ont été publiées [24, 25]. Elles sont aujourd'hui complétées par des études plus quantitatives associées à des modélisations 
micro-mécaniques de type plasticité cristalline [26]. Du point de vue des mécanismes physiques, il est probable que dans ces alliages, des effets de l'hydrogène sur l'ordre à courte distance soient à prendre en compte.

Dans les environnements qui présentent une activité faible ou moyenne de l'hydrogène, les aciers inoxydables austénitiques présentent généralement une rupture ductile à cupules avec un allongement et une striction réduites. De ce point de vue, le phénomène précité de localisation accrue de la déformation fournit une explication directe de la réduction de ductilité, et les études en cours sont prometteuses quant à la perspective d'une modélisation prédictive. Les ruptures intergranulaires fragiles (cas de la majorité des alliages à base de nickel) nécessitent, elles, la prise en compte d'effets de co-ségrégation entre espèces fragilisantes et hydrogène aux joints de grains et/ou joints de phases [23]. Des travaux complémentaires basés sur des matériaux de pureté contrôlée et l'utilisation de moyens modernes d'analyse des surfaces doivent permettre de progresser dans la quantification de ces phénomènes. Les résultats expérimentaux récents sur les alliages Al-Mg démontrent par ailleurs que la plasticité «incidente» sur les joints de grains joue un rôle important dans la rupture intergranulaire semi-fragile par Corrosion Sous Contrainte [27]. D’une manière générale, la prise en compte de la plasticité à l'échelle des lignes de glissement individuelles à l'extrême voisinage des fissures est nécessaire pour modéliser la rupture «fragile» des matériaux c.f.c. par effets de l'environnement. Si la faible solubilité de l'hydrogène dans les alliages d'aluminium permet de supposer que la prise en compte des effets de couplage décrits dans cet article n'est peut-être pas nécessaire en première approche dans ces matériaux, il en va autrement des cas de rupture transgranulaire fragile dans les aciers austénitiques qui sont observés en présence d'une forte activité de l'hydrogène : la solubilité de l'hydrogène est élevée dans ces alliages et il existe de nombreuses preuves expérimentales de l'importance des effets de couplage entre hydrogène et déformation plastique dans ces processus de rupture [28]. Nous développons à cet effet une simulation bidimensionnelle de dynamique des dislocations en pointe de fissure [29]. Les premiers résultats montrent notamment l'importance de disposer d'un critère physique d'émission homogène des dislocations à la pointe de fissure pour reproduire une dynamique d'écrantage réaliste. Une modélisation à l'échelle atomique des effets de l'hydrogène sur cette émission homogène constitue une première étape importante pour la prise en compte de ces effets sur la rupture fragile. Concernant de possibles effets couplés dans la zone plastique environnante, les résultats de modélisation présentés dans cette communication suggèrent comment il sera possible à l'avenir d'incorporer les effets de l'hydrogène dans une telle modélisation.

\section{Références}

[1] Birnbaum H.K., Robertson I.M., Sofronis P. and Teter D. "Mechanisms of hydrogen related fracture : a review", Second International Conference on Corrosion- Deformation Deformation, Nice, France, 1996, Magnin T. Ed. (The Institute of Materials 1997) pp. 172-195.

[2] Gangloff R.P., "Critical issues in hydrogen assisted cracking of structural alloys". EnvironmentInduced Cracking of Materials, Banff, Alberta, Canada, 2004, Shipilov S.A., Jones R.H., Olive J.M., Rebak R.B. Eds (Elsevier Science, 2008) 141.

[3] Brass A.M. and Chene J. Mat. Sci. Eng. A 242 (1998) 210.

[4] Brass A.M. and Chene J., Corr. Sci. 48 (2006) 3222.

[5] Birnbaum H.K. and Sofronis P., Mat. Sci. Eng. A 176 (1994) 191.

[6] Chene J. and Brass A.M., Scripta Mat. 40 (1999) 537.

[7] Wilcox B.A. and Smith G.C. Acta Metall. 12 (1964) 371.

[8] Combette P. and Grilhé J, Mémoires Scientifiques de la Revue de Métallurgie, LXVII (1970) 491-505.

[9] Girardin G. "Interactions hydrogène - plasticité dans le nickel et ses alliages", Thèse de doctorat, Saint-Etienne: Ecole Nationale supérieure des Mines et Université Jean Monet (2004)

[10] Kubin L.P, Estrin Y. Acta Metall. Mater. 35 (1990) 697. 
[11] Sofronis P., J. Mech. Phys. Solids. 43 (1995) 1385.

[12] Chateau J.P. "Vers une quantification des mécanismes de corrosion sous contrainte : simulations numériques des interactions hydrogène - dislocations en pointe de fissure", Thèse de doctorat, Saint-Etienne : ENSM.SE - INPG (1999).

[13] Chihab K, Estrin Y, Kubin L.P. and Vergnol J, Scripta Met. et Mat. 21 (1987) 203.

[14] Fressengeas C., Beaudoin A.J., Lebyodkin M., Kubin L.P. and Estrin Y., Mat. Sci. Eng. A 400-401 (2005) 226.

[15] Girardin G. and Delafosse D. Scripta Mater. 51 (2004) 1177.

[16] De A.K., Vandeputte S., De Cooman B.C. Scripta Mater. 41 (1999) 831.

[17] Larché F., Cahn J.W. Acta Metall. 33 (1985) 331.

[18] Sofronis P., Birnbaum H.K, J. Mech. Phys. Solids. 43 (1995) 49.

[19] Chateau J.P, Delafosse D. and Magnin T., Acta Mater. 50 (2002) 1507.

[20] De Wit G, Koehler J.S. Phys. Rev. 116 (1959) 1113.

[21] Wen M., Fukuyama S., Yokogawa K., Scripta Mat., 52 (2005) 959.

[22] Yagodzinskyy Y., Tarasenko O. and Hänninen H. "Effect of hydrogen on plastic deformation of stable 18Cr-16Ni-10Mn austenitic stainless steel single crystals", Hydrogen Effects on Materials and Corrosion Deformation Interactions, Moran W.Y 2002, Moody N.R., Thompson A.W., Ricker R.E., Was G.W., Jones R.H. Eds (TMS, Warrendale, PA, 2003) pp. 201-210

[23] Lassila D.H. and Birnbaum H.K. Acta Metall. 35 (1987) 1815.

[24] Ulmer D.G., Altstetter C.J., "Mechanism for Hydrogen Embrittlement of Austenitic Stainless Steels", Hydrogen Effects on Materials Behavior, Moran W.Y. 1989, Moody N.R., Thompson A.W. Eds (TMS, Warrendale PA 1990) pp. 421-430.

[25] San Marchi C., Somerday B.P., Tang X., Schiroky G.H. Int. J. of Hydrogen Energy, 33 (2008) 889.

[26] Aubert I., Plessier F., Saintier N., Olive J.M. (2008) "Modification of plastic strain localization induced by hydrogen absorption, Advances in Materials Science", Vol. 8, issue 1, in press.

[27] Tanguy D., Bayle B., Dif R., Magnin T., Corr. Sci. 44 (2002) 1163.

[28] Chambreuil-Paret A., Chateau J.P., Magnin T., Scripta Mater. 37 (1997) 1337.

[29] Tanguy D., Razafindrazaka M., Delafosse D., Acta Mater. 56 (2008) 2441. 
